\title{
Meta
}

Journal des traducteurs

Translators' Journal

\section{Heterolingüismo y autotraducción. El caso de Álvaro Cunqueiro}

\section{Rexina Rodríguez Vega}

Volume 60, numéro 1, avril 2015

URI : https://id.erudit.org/iderudit/1032399ar

DOI : https://doi.org/10.7202/1032399ar

Aller au sommaire du numéro

Éditeur(s)

Les Presses de l’Université de Montréal

ISSN

0026-0452 (imprimé)

1492-1421 (numérique)

Découvrir la revue

Citer cet article

Rodríguez Vega, R. (2015). Heterolingüismo y autotraducción. El caso de Álvaro Cunqueiro. Meta, 60(1), 36-52. https://doi.org/10.7202/1032399ar
Résumé de l'article

À partir de l'analyse des autotraductions d'Álvaro Cunqueiro, un auteur appartenant au canon de la littérature galicienne et espagnole, nous étudions les éventuelles implications du bilinguisme endogène dans l'autotraduction. On constate ainsi une nette augmentation de la tension entre la tendance à la préservation littérale et littéraire de l'hétérolinguisme et, d'autre part le désir d'acceptabilité dans la culture d'arrivée. Ces deux forces opposées, exacerbées à l'extrême par un autotraducteur qui se sent libéré du fantôme de l'invisibilité engendrent, finalement, un texte trop hétérogène, marqué par des stratégies énonciatives variées qui, en même temps, laissent transparaître clairement une voix personnelle et « différente » de l'autotraducteur comme locuteur second. 


\title{
Heterolingüismo y autotraducción. El caso de Álvaro Cunqueiro
}

\author{
REXINA RODRÍGUEZ VEGA \\ Universidade de Vigo, Vigo, España \\ xinavega@uvigo.es
}

\section{RÉSUMÉ}

À partir de l'analyse des autotraductions d'Álvaro Cunqueiro, un auteur appartenant au canon de la littérature galicienne et espagnole, nous étudions les éventuelles implications du bilinguisme endogène dans l'autotraduction. On constate ainsi une nette augmentation de la tension entre la tendance à la préservation littérale et littéraire de l'hétérolinguisme et, d'autre part le désir d'acceptabilité dans la culture d'arrivée. Ces deux forces opposées, exacerbées à l'extrême par un autotraducteur qui se sent libéré du fantôme de l'invisibilité engendrent, finalement, un texte trop hétérogène, marqué par des stratégies énonciatives variées qui, en même temps, laissent transparaître clairement une voix personnelle et «différente» de l'autotraducteur comme locuteur second.

\section{ABSTRACT}

Based on the analysis of self-translation done by Álvaro Cunqueiro, a recognized author in both Galician and Spanish literature, possible implications of endogenous bilingualism in self-translation are studied. One observes increased tension between the trend of literal and literacy preservation of heterolingualism and the desire to be accepted in the target language. These two competing forces, taken to the extreme by an author-translator freed from the spectre of invisibility, ultimately offer an excessively heterogeneous text, marked by expository strategies of a diverse nature which, at the same time, clearly show the "unique" voice of the self-translator as a second interlocutor.

\section{RESUMEN}

A partir del análisis de las autotraducciones de Álvaro Cunqueiro, un autor reconocido por el canon tanto en la literatura gallega como en la castellana, se estudian las posibles implicaciones del bilingüismo endógeno en la autotraducción. Así se observa cómo se acentúa la tensión entre la tendencia a la preservación literal y literaria del heterolingüismo y el deseo de aceptabilidad en la cultura meta. Estas dos fuerzas opuestas, llevadas al extremo por un autor-traductor liberado del fantasma de la invisibilidad, acaban por ofrecer un texto excesivamente heterogéneo, marcado por estrategias enunciativas de signo diverso que, al mismo tiempo, manifiestan con claridad la voz propia y "distinta" del autotraductor como locutor segundo.

\section{MOTS-CLÉS/KEYWORDS/PALABRAS CLAVE}

autotraduction, bilinguisme endogène, hétérolinguisme, bilittéralité, énonciation self-translation, endogenous bilingualism, heterolingualism, interliterary process, enunciation autotraducción, bilingüismo endógeno, heterolingüismo, biliterariedad, enunciación 


\section{Introducción}

Como indica Julio César Santoyo (2010: 365-366), la autotraducción ha sido, hasta hace muy poco, considerada como un ejercicio raro y poco relevante en los estudios de traducción. De hecho, hay que esperar hasta los albores del nuevo milenio para que comiencen a aparecer trabajos centrados en el peculiar y, en realidad, dilatado y universal fenómeno de la traducción llevada a cabo por el propio autor. Así, además de los estudios, en cierta medida inaugurales, de Brian Fitch (1983; 1985), Michaël Oustinoff (2001; 2003) o Rainier Grutman (1997; 2007; 2009; 2011), debe señalarse el interés creciente que suscita el análisis de este fenómeno en el ámbito de las literaturas ibéricas, en el que destacan los trabajos de Helena Tanqueiro (1999; 2002), Patricia López Gay (2008), Francesc Parcerisas (2007; 2009; 2011) o Xosé Manuel Dasilva (2009; 2010; 2011; 2012).

Aunque el espacio de la península ibérica cuenta con una larguísima tradición tanto de bilingüismo de escritura como de autotraducción (Santoyo 2010) será, sobre todo, en la España posfranquista cuando este fenómeno alcance su apogeo. Y es que, de acuerdo con un patrón de intercambio desigual, son, fundamentalmente, los autores de la literatura gallega, catalana y vasca quienes frecuentemente traducen sus obras a la lengua mayoritaria. Este tipo de trasvase, definido por Grutman (2011) como "supraautotraducción" conlleva a menudo aparejada también la tendencia a la "recreación", práctica que pone en cuestión el concepto mismo del texto original.

Además de esta interrogante fundamental respecto al estatuto del nuevo texto, las autotraducciones en el ámbito de las literaturas hispánicas manifiestan, en mayor o menor grado, la presencia de un hibridismo de códigos o "heterolingüismo", entendido como una consecuencia, hasta cierto punto inevitable, del "bilingüismo de escritura endógena” (Grutman 2007), es decir, el propio de aquellos autores que manejan lenguas en contacto en situación diglósica.

Es mi intención en el presente trabajo abordar el caso de un autor, Álvaro Cunqueiro Mora, que considero paradigmático por cuanto ilustra de modo especialmente nítido las tensiones que pueden producirse en el ejercicio de la autotraducción en el contexto peninsular.

Cunqueiro, escritor monolingüe en gallego en poesía ${ }^{1}$ y teatro y claramente bilingüe en el ámbito de la novela y el periodismo literario, presenta la particularidad de haber autotraducido una buena parte de su obra narrativa del gallego al castellano. Podemos citar, entre otros títulos, Merlín e Familia e outras historias (1955) / Merlín y familia (1957), As crónicas do Sochantre (1956) / Las crónicas del Sochantre (1959)2, Si o vello Sinbad volvese ás illas (1961) / Cuando el viejo Sinbad vuelva a las islas (1962) o Xente de aquí e acolá (1971) / La otra gente (1975) ${ }^{3}$.

Nuestro análisis partirá del cotejo entre estas versiones, abordándolas tanto desde el punto de vista del trasvase lingüístico y cultural como desde las transformaciones ficcionales que presentan.

\section{Heterolingüismo y condiciones de enunciación}

Desde el punto de vista de las condiciones de producción, del contexto comunicativo en el que se inscribe el texto autotraducido, es relevante llamar la atención sobre la confluencia en la identidad del locutor que produce el discurso en una y otra lengua. 
La autotraducción se distingue así por la existencia de una distancia cero, en términos de subjetividad, entre autor y traductor, dado que ambas funciones son asumidas por la misma persona.

Este particular estatus debería, como asegura Tanqueiro (1999), dar como resultado la "invisibilidad" extrema del traductor. Sin embargo, dicho enfoque se me aparece como excesivamente simplificador. El heterolingüismo, definido por Grutman como "la présence dans un texte d'idiomes étrangers, sous quelque forme que ce soit, aussi bien que des variétés (sociales, régionales ou chronologiques) de la langue principale" (Grutman 1997: 37), es una constante en las autotraducciones cunqueirianas, y, también, en las de buena parte de las autotraducciones en condiciones de bilingüismo de escritura endógena. Esa clara tendencia resulta, en mi opinión, reveladora de la existencia de una clara visibilidad del autotraductor como locutor segundo en el que es posible distinguir una "voz" propia y "distinta".

En cierta medida, la autotraducción se constituye como un campo de pruebas privilegiado para refrendar la tesis planteada por Myriam Suchet (2009), quien reacciona contra el mito de la invisibilidad del traductor oponiendo el cambio de código como uno de los indicios más reveladores de la necesaria existencia de una estrategia enunciativa a la que la investigadora se refiere empleando el término clásico de ethos. Así, para Suchet: "La fonction principale de l'hétérolinguisme est de construire une image de l'instance énonciatrice. Reconduite sur le terrain de la traduction, cette critique de l'illusoire invisibilité de l'énonciateur permet de faire de la notion d'ethos la base d'un modèle de l'énonciation caractéristique de la traduction" (Suchet 2009: 2).

A continuación, y siguiendo la taxonomía de Suchet, rastrearemos las diferentes estrategias que, en relación con el heterolingüismo, presentan las autotraducciones de Cunqueiro.

\subsection{Ethos de mediación explícita}

El ejercicio cunqueiriano acostumbra a manifestar una concepción de la traducción como un espejo en el que se refleja la realidad original sin excesivos filtros, asumiendo las mismas condiciones enunciativas en los dos textos y, por lo tanto, renunciando a adaptar referencias espaciales o culturales específicas. La consecuencia de esta postura será la frecuente inclinación del Cunqueiro traductor a dejar paso a la voz gallega del original; pero no siempre la presencia de los galleguismos proviene de la misma modalidad de asunción de las condiciones de enunciación en el texto traducido. A veces, como sucede en el libro La otra gente, el Cunqueiro traductor asume el papel de intermediario haciendo de puente entre dos universos referenciales diferentes. En estos casos, Cunqueiro opta por allanar las dificultades de recepción sin renunciar a convocar en el texto determinadas voces gallegas características de la realidad que pretende comunicar. Así, el procedimiento habitual será el de ofrecer la equivalencia castellana acompañada de una glosa en la que se da el término original. La peculiaridad de esta situación, en la que el narrador-traductor declara su pertenencia al universo referencial del texto original, sin dejar por ello de actuar como eficaz intermediario cultural, es posible en la medida en la que existe una identificación entre las voces de narrador, autor y traductor. De hecho, esta triple dimensión del locutor del texto de llegada es consecuencia directa de la configuración de la voz narrativa del texto de partida. En Xente de aquí e acolá, así como en los otros volúmenes inte- 
grantes de lo que se dio en denominar la "trilogía de relatos gallegos" Escola de menciñeiros y Os outros feirantes, se observa cómo el propio Cunqueiro, ficcionalizado como narrador, aprovecha la fuerza realista que se deriva de la apariencia biográfica para crear un mundo fabulado. Por lo tanto, a la hora de abordar la versión al castellano es lógico o hasta cierto punto esperable que el narrador, identificado con el autor, introduzca comentarios que revelan la consciencia del cambio de destinatario que se produce en la traducción. La función didáctica o divulgativa que cumplen estas glosas incide en la intensificación de determinados rasgos temáticos que caracterizaban al texto original. Y es que si en Xente de aquí e acolá nos encontramos con un ejercicio de recreación de la vida rural gallega trascendido por la imaginación creadora de Cunqueiro, en La otra gente la dimensión etnográfica y antropológica parece acentuarse debido a comentarios puramente informativos o documentales.

(1) O golpe falaba mui ben o galego, e co acento de alí mesmo, onde dicen autro por outro e aira por eira.

(Xente: 46)

(a) El zorro, raposo o golpe, como le decimos los gallegos, hablaba muy bien nuestra lengua, y con el acento de aquella misma comarca, donde dicen autro por outro, otro, y aira por eira, era.

(Gente: 163)

(2) E as palletas da gaita súa eran italianas.

(Xente: 134)

(a) Las lengüetas de su gaita -los gallegos les llamamos "palletas"-eran italianas.

(Gente: 150)

Como podemos comprobar aquí, la presencia del heterolingüismo hace audible al autor en su papel de mediador al tiempo que dibuja una imagen del lector implícito. Esta tendencia, más común de lo que pudiese parecer, se erige, de hecho, en una característica de muchas de las autotraducciones peninsulares en las que la nota o la glosa aparecen incorporadas en el cuerpo mismo del texto alterando su estatus primigenio. En la base de este fenómeno se halla, a mi juicio, la particular concepción de "extranjería" de las lenguas peninsulares cooficiales, un grado de distancia "menor" que supone, de hecho, una aceptación tácita de su pertenencia a un espacio cultural común.

\subsection{Ethos de obra}

Pero la presencia de galleguismos en la versión castellana de Cunqueiro puede obedecer también a otro tipo de estrategia enunciativa. Probablemente, la mayor parte de las manifestaciones del heterolingüismo en las autotraducciones de este autor se enmarquen dentro de lo que Suchet denomina "ethos d'ouvre" en el que la alternancia de códigos funcionaría como "un rite de passage vers un emploi non quotidien du langage" (Suchet 2009: 11).

Esta función, relacionada con el "effet de réel" de Barthes y el "effet d'œuvre" propuesto por Grutman (1997: 44) indica la entrada de la enunciación en un régimen a la vez literario y literal que no puede más que enfatizar el carácter hipertextual de la versión.

El predominio de la literalidad demuestra en Cunqueiro un ejercicio consciente de descentramiento de los usos lingüísticos de la lengua/cultura receptora. La tra- 
ducción se convierte así en un ejercicio donde la fidelidad y la libertad se unen con el objetivo de la reproducción, de la mímesis, de características estructurales del gallego que puedan ser absorbidas sin excesiva violencia por el castellano.

La condición de bilingüe endógeno del autor-traductor opera, sin duda, como factor determinante en este empleo del heterolingüismo. Tal y como advierte Steiner (1995: 266-268) -compartiendo en cierta medida la misma concepción del literalismo que alimenta las líneas teóricas de Meschonnic (1973; 1989) o Berman (1985)- la incursión hermenéutica, el impulso de comprensión entre ámbitos muy próximos - como es el caso del gallego y el castellano- se realiza a través de círculos concéntricos de conciencia lingüística y cultural, de información probable y de identificación. Estos círculos concéntricos generan criterios de comparación y analogía mediante los que es posible evaluar el grado de comprensión y de posible transferencia, al mismo tiempo que esclarecen y explicitan el texto fuente. Son precisamente esos mismos círculos los que vuelven también al texto por traducir más denso y más opaco. Las relaciones del traductor con lo que está cerca de él son pues, por definición, ambiguas y dialécticas, determinadas como están por la presencia simultánea de la afinidad electiva y de la diferencia resistente.

Así, según apunta Steiner, la vivencia de la diferencia es mucho más intensa desde una relación de proximidad. El traductor se ve obligado a actualizar y volver visibles los perímetros de su lengua, de su cultura, de su carga potencial de sensibilidad e intelectualidad. A través de una red de analogías, de metafrases, de locuciones híbridas se produce una reflexión y una readquisición del idioma propio sobre el que influye, para iluminarlo, la lengua del texto original.

Este proceso tiende, como esperamos demostrar en este artículo, a intensificarse hasta el extremo en el caso de un autotraductor como Cunqueiro. La vivencia de la duplicidad y el proceso mismo de reflexión que conlleva la operación del trasvase idiomático genera una traducción "descentrada” (Meschonnic 1973) en la que la preservación de la extranjería del original sirve como vía de experimentación y desautomatización de usos lingüísticos en la lengua de llegada, convirtiéndose así en un eficaz motor estilístico.

Entre las manifestaciones más destacadas del trabajo lingüístico fronterizo que desarrolla Cunqueiro destacaría, por ejemplo, la creación neológica producto de la tradución literal:

(3) [...] e cando ao mencer ou á tardiña lle dá o sol escorado entón arroiba

(Sinbad: 430)

(a) $[\ldots]$ y cuando al amanecer o anocheciendo le da el sol escorado, entonces arrubia

(Sinbad-b: 136)

(4) [...] un lobo vello, dos que chaman por alá "garlines", porque non deixan nunca a ronda dos lugares i aldeas e destemen ao home [...]

(Merlín: 54)

(a) un lobo viejo, de los que llaman por allá "garlines", porque no dejan nunca la ronda de los lugares y aldeas y destemen al hombre

(Merlín-b: 56)

(5) [...] e os parlantes [os peixes-papagaio] ruben á tona do mar

(Sinbad: 366)

(a) [...] y los bien parlantes suben a la nata del mar

(Sinbad-b: 72) 
La reproducción de locuciones y modismos del gallego que dan lugar a sintagmas no lexicalizados en castellano:

(6) [...] parez que dorme profundo, polo que ronca, pro se si alguén se acaroa á caixa dos cartos, por mui pés de lá que o faga, cando xa está a duas varas o Cangrexo abre o ollo esquerdo e cuspe o fío da baba.

(Sinbad: 372)

(a) [...] parece que duerma profundo, por lo que ronca, pero si alguien se acerca a la caja de los dineros, por muy pies de lana que traiga, cuando ya está a dos varas el Cangrejo abre el ojo izquierdo y escupe el hilo de baba.

(Sinbad-b: 78)

(7) ¡Eses califatos pensan que non hai máis que chegar i encher!

(Sinbad: 346$)$

(a) ¡Estos califatos creen que no hay más que llegar y llenar!

(Sinbad-b: 53)

O la alteración del orden de los elementos en la oración que reproduce la tendencia hiperbática de las construcciones gallegas como medio para la expresión del énfasis:

(8) E díxenllo ao Mirán, que é como se chama El-Rei dacolá, cando lle fun vender o peixe-papagaio, que nunca outro se pescou máis que aquel

(Sinbad: 365)

(a) Y se lo dije al Mirán, que es como se llama al rey de allí, cuando le fui a vender el pez papagayo, que nunca otro se pescó más que aquél

(Sinbad-b: 70)

(9) Foi iste don Esmeraldino vizconde, polo que dil teño escoitado a un seu criado de librea i escopeteiro

(Merlín: 123)

(a) Fue este don Esmeraldino Vizconde, por lo que de él oí contar a un su criado de librea y escopetero

(Merlín-b: 139)

Como vemos, el hibridismo lingüístico surge aquí de un ethos que busca preservar la significancia del texto, de acuerdo con un modelo de traducción que tiende a reproducir los valores expresivos de la lengua del original, transformando así la lengua de llegada bajo la presión de lo "diferente".

Dentro de las manifestaciones del heterolingüismo en las versiones cunqueirianas quizás deba tratarse como un aspecto específico la dimensión arcaizante que confiere al castellano la influencia del gallego como lengua de fondo. Así podemos comprobar cómo frecuentemente nuestro escritor tiende a privilegiar en la traducción aquellas formas coincidentes en las dos lenguas, pero sin equivalencia sincrónica.

Surgidos de la traducción literal de la forma gallega, los numerosos arcaísmos que presentan las versiones castellanas pueden, a nuestro juicio, cumplir diferentes funciones. En primer lugar, es necesario preguntarnos aquí si su empleo obedece a una intensificación consciente de la galleguidad del texto o a un simple fenómeno de interferencia lingüística. En todo caso, el receptor de la autotraducción procederá frecuentemente a identificar determinados usos como propios del uso regional del castellano en Galicia. 
Esto es lo que sucede, por ejemplo, con el abundante uso del pluscuamperfecto etimológico:

(10) Jacinto tenía una lengua muy larga, que le revertía de la boca cuando estaba distraído, y que le valiera muchos arrestos cuando hizo el servicio militar en Zamora 8, en Lugo. Y ahora, desde que era paraguas o habitaba el paraguas, aún le creciera más con el ejercicio que hacía.

(Gente: 48)

Más interesantes resultan, sin duda, aquellos arcaísmos que evidencian una clara intencionalidad estilística. Cunqueiro parece aquí servirse de la influencia ejercida sobre el castellano desde la lengua gallega para potenciar la atmósfera de distanciamento y atemporalidad que conviene a su mundo novelesco.

Así se percibe en el uso de determinadas unidades léxicas:

(11) E con aquel xogo de luces, a porta pechada, decirlle a Alba querida que erguera o faldellín, e despoixas a sobresaia, e despoixas a saia

(Sinbad: 380)

(a) Y con aquel juego de luces decirle a Alba querida que levantara el faldellín, y después la sobresaya, y después la saya

(Sinbad-b: 86)

(12) [...] naide ousase usar aquel invento nos seus xantares

(Sinbad: 348)

(a) [...] nadie osase usar aquel invento en sus yantares

(Sinbad-b: 56)

(13) [...] sentado no sillón de veludo verde

(Merlin: 21)

(a) [...] sentado en el sillón de velludo verde

(Merlín-b: 21)

o en construcciones como las que siguen:

(14) O gran xefe dos lugueses miróuno con aqués seus ollos vivos e bulrós

(Xente: 27)

(a) El gran jefe de los lugueses lo miró con aquellos sus ojos vivos y burlones

(Gente: 27)

(15) [...] e que o señor Merlín, que era moito seu amigo

(Merlín: 35)

(a) [...] y que el señor Merlín, que era muy su amigo

(Merlín-b: 35)

(16) A mi amo gostáronlle moito as historias de Elimas, mercóulle sete libros

(Merlín: 55)

(a) A mi amo le gustaron mucho las historias de Elimas, compróle siete libros

(Merlín-b: 58)

Como podemos comprobar, el recurso a la traducción literal sirve a menudo a Cunqueiro para dotar su castellano de un aroma arcaizante y marcadamente literario. Lo que en el original formaba parte del uso normal del habla se convierte en la 
versión castellana en una expresión literaturizada que incurre, a veces, en el cliché retórico. Este hecho evidencia hasta qué punto difiere el trabajo estilístico de nuestro autor en una u otra lengua. La predilección por formas pertenecientes a un modelo de lenguaje literario hipercodificado que caracteriza el castellano de Cunqueiro parece, pues, deberse tanto a una influencia del sistema gallego como a una voluntad expresa de potenciar la imitación estilística de la tradición clásica española. En este sentido resultan reveladores los tipos de subgéneros: novela de caballerías, sentimental, pastoril... sobre los que construye sus parodias en las novelas redactadas originariamente en castellano.

El estudio de las manifestaciones del heterolingüismo en Cunqueiro constituye pues, en mi opinión, una excelente vía para el análisis del complejo fenómeno de la autotraducción. La confluencia del traductor con el autor no tiene por qué tener como efecto la invisibilidad máxima del trabajo del mediador; al contrario, esta circunstancia puede poner de relieve con una fuerza inusitada, tal y como sucede en el caso que nos ocupa, la existencia del traductor entendido como un enunciador segundo que posee su propia voz. El carácter dialógico de este proceso se vuelve aún más intenso si tenemos en cuenta, además, la implicación que el desdoblamiento tiene en el proyecto global de escritura del autor.

\subsection{Ethos de obra y "asimilación"}

Tal y como antes indicábamos, siguiendo a Steiner, la vivencia de culturas próximas es, por definición, ambigüa y dialéctica. Por este motivo no solo la tendencia al descentramiento y la disimilación se erige como una característica habitual en la autotraducción. Así, en el caso de Cunqueiro, encontramos también el justo correlato de acuerdo a un "ethos d'œuvre" que busca amoldarse a las normas de la cultura meta, conforme con un modelo de traducción que lleva al límite la tendencia a la "aceptabilidad” (Toury 1980) y que, por lo tanto, supone la eliminación de las manifestaciones del heterolingüismo en el texto de llegada.

Los estudios sobre el bilingüismo en la creación literaria advierten de la frecuente inclinación de los escritores a demostrar un dominio excéntrico y exhibicionista de la lengua no materna ${ }^{4}$. Este fenómeno, que se produce no solo en los creadores bilingües, quienes, en realidad, acostumbran a ejercer una suerte de "metatraducción", sino también en los simples intermediarios culturales, da como resultado la obtención de un texto más normativo de lo que sería esperable en una obra escrita directamente en la lengua de llegada. El resultado, pues, de esta inclinación, será el desarrollo de un proceso de reescritura en el que interviene masivamente la literaturización e incluso, como indica el teórico francés Berman (1985: 54), la "sobre-literatura".

En su análisis sobre las tendencias deformantes que se producen en la traducción literaria, Berman señala la existencia de categorías como el anoblissement y la homogénéisation. Mediante el anoblissement tiene lugar una reescritura del original atenta a la consecución de un texto formalmente más hermoso. La búsqueda de la legibilidad, la eliminación de los focos de oscuridad, ambigüedad o presunta torpeza, la intensificación de los elementos retóricos, literaturiza el original a costa de su inevitable banalización. Como complementaria y resultante de esta tendencia ennoblecedora, la homogénéisation es definida por Berman como una operación que "consiste à unifier sur tous les plans le tissu de l'original, alors que celui-ci est originairement 
hétérogène" (Berman 1985: 75). La homogeneización supondría, por lo tanto, la destrucción de las redes significantes subyacentes, de las sistematicidades, de los ritmos, de las locuciones, de la superposición de las lenguas y de las redes de lenguajes vernáculos.

Ambos procesos, "ennoblecimiento" y "homogeneización”, pueden rastrearse claramente en la obra de Cunqueiro. Tanto en los originales escritos directamente en castellano como en la autotraducción de los originales gallegos, la lengua y el estilo del autor aparece marcado por una tensión que lo lleva a exhibir un poderío verbal, no pocas veces artificioso y convencional.

Así, frente al impulso hacia la literalidad que potencia las manifestaciones del heterolingüismo, podemos observar una estrategia enunciativa de signo radicalmente opuesto en la que el juego de fuerzas entre el registro culto y el popular se difumina, neutralizando la heteroglosia del texto de partida.

Pensemos, por ejemplo, en la sustitución de la expresividad de voces, locuciones y modismos propios del ámbito de la oralidad, por soluciones estándar:

(17) [...] e púxose consigo mesmo en que había de petar pra riba

(Sinbad: 370)

(a) [...] que se puso consigo mismo en que había de crecer

(Sinbad-b: 76)

(18) [...] e estando no millor, vai e parou, e axoenllóuse

(Xente: 20)

(a) [...] y estando en lo mejor y más deleitoso del trabajo, se detuvo y se arrodilló.

(Gente: 20)

o, dentro de una clara maniobra de compensación estilística, por fórmulas propias del lenguaje literario:

(19) [...] e no patín do Firí volvía a muxíla burra, bebía o leite quentiño

(Sinbad: 385)

(a) [...] y en el patio del Firí volvía a ordeñar la burra, bebía la tibia leche

(Sinbad-b: 91)

(20) $[\ldots]$ co aquel seu montar escarranchado

(Merlín: 58)

(a) [...] con aquel su abierto y reposado montar

(Merlín-b: 59)

(21) [...] mirando prá xente de bullanda

(Merlín: 116)

(a) [...] viendo el animado y abigarrado concurso

(Merlín-b: 128)

La supresión de las marcas de la oralidad se refleja también en la mayor precisión léxica, que, en ocasiones, da por resultado una fuerte incoherencia en la configuración del narrador homodiegético, a quien se le atribuye una competencia lingüística que, como en el caso del criado campesino del Merlín, Felipe de Amancia, no resulta verosímil: 
(22) Trouxen a leite, e dóulla o señor Merlín a cullaradas ao animaliño aquel

(Merlín: 41)

(a) Porté la leche, y se la dio don Merlín a cuharaditas al animalito aquel

(Merlín-b: 43)

Así mismo, se observa un proceso de transformación de las estructuras sintácticas de acuerdo a las pautas del discurso escrito. La eliminación de los elementos anafóricos o la reorganización de los enunciados suprimiendo o diversificando los conectores argumentativos son algunas de las manifestaciones de este proceso:

(23) Axuntóuse en Termar meia compañía de bernardos de Meira, e toda a dos caseiros e criados por ver o galo don Esmeraldino, que era, xa dixen, unha fermosura de cantacraro

(Merlín: 127)

(a) Se reunió en Termar media compañía de bernardos de Meira y toda la de los caseros y criados por ver el gallo don Esmeraldino, que era una hermosura de cantaclaro

(Merlín-b: 144)

(24) Pro don Silvestre non puido dar un paso, que metéu o pe esquerdo no cepo, e cantou deseguida o sino de prata e mi amo berrou non sei que latín, i eu corrín con doña Simona ao seu amparo, i esbaramos ao chegar á fonte, caímos no lameiro, i eu priveime....

(Merlín: 45-46)

(a) Pero don Silvestre no pudo dar un paso, que metió el pie izquierdo en el cepo, y cantó en seguida la campanita de plata, mi amo gritó no sé qué latín, yo corrí con doña Simona a su amparo, pero resbalamos al llegar a la fuente, caímos en el lodo, y yo me desmayé...

(Merlín-b: 49)

En otros casos se produce una amplificación retórica a partir, y a costa, del original:

(25) Falo das luces que andaban polos camiños [...] e de cando en veces xuntábanse tres ou catro i el era como unha pequena fogueira no medio da noite

(Merlin: 15)

(a) Hablo de las luces que andaban por los caminos [...] y de cuando en cuando se cruzaban tres o cuatro, que hacían como una pequeña hoguera en el corazón de la oscura noche

(Merlín-b: 15)

(26) o camiño ha de labrar os seus pasos [...] pra poder logo botarse pola chaira de Beiral, que se tende á destra das carballeiras bernardas, coa xentileza das abidueiras ollándose nas lamas

(Merlín: 110)

(a) el camino ha de labrar sus pasos [...] para poder luego tenderse feliz por la llanura de Beiral, donde son las abiertas veranias, el coro solemne de las robledas bernardas, y la gentileza de los abedules mirándose estremecidos en las quietas charcas.

(Merlín-b: 118) 


\section{La autotraducción como reescritura: las transformaciones textuales}

Dentro del análisis comparativo entre los originales gallegos y las versiones castellanas realizadas por el propio escritor, sobresalen por su importancia aquellas modificaciones que implican una seria alteración del universo ficcional, lo que obliga, inevitablemente, a poner en cuestión el concepto de texto original.

Fitch señaló como diferencia fundamental entre la traducción alógrafa y la traducción de autor el hecho de que, frente al carácter metatextual de la primera, la segunda puede constituir una creación artística autónoma (Fitch 1983: 89); Oustinoff, por su parte, habla de "segundo original", diferenciando dentro de los diversos tipos de autotraducción aquella a la que denomina "auto-traduction (re)créatrice" (Oustinoff 2001: 33-34).

Sin duda, la obra autotraducida por Cunqueiro entraría de lleno en esta última categoría. Las alteraciones que presentan las versiones castellanas son fruto de la aplicación de procedimientos de ampliación que van desde la esfera del detalle hasta el añadido de contenidos ficcionales que modifican la configuración general del texto. Así pues, podríamos determinar dos vías fundamentales para la transformación: la expansión estilística y la extensión temática.

Las expansiones estilísticas que se observan en el cotejo evidencian el trabajo de corrección del autor que, enfrentado de nuevo a su texto, tiende a actualizar las potencialidades que en el original quedaban latentes. La caracterización más detallada de objetos, personajes y situaciones tiene pues como objetivo la intensificación de las estrategias realistas. En otros casos la ampliación estilística afecta a la misma estructura del relato. Se da aquí una tendencia hacia la clarificación y la normalización del texto de acuerdo a un modelo más canónico. Esta operación se manifiesta, sobre todo, en el volumen de relatos breves La otra gente, donde la reelaboración supone una pérdida de los valores orales del texto original a favor de la contención y perfeccionamiento estructural propios del discurso escrito.

Pero son, sin duda, las modificaciones por extensión temática las de mayor repercusión en tanto que suponen un importante grado de desviación respecto al texto gallego. Así en Merlín y familia encontramos el añadido de "Pablo y Virginia" y "Noticias varias de la vida de don Merlín, mago de Bretaña"; en Cuando el viejo Sinbad vuelva a las islas, del "Retrato de la viuda Alba" y del "Índice onomástico" y en Las Crónicas del Sochantre, del "Epílogo para Bretones".

Es importante señalar aquí el hecho de que, frente a los modelos novelísticos cerrados, homogéneos y globales, Cunqueiro opone una obra caracterizada por la apertura y la desestabilización textual, por lo tanto en esta propuesta narrativa el peritexto juega un importante papel. Así, uno de los recursos más empleados por el autor consiste en subvertir el texto central mediante el añadido de un apéndice que ofrece una nueva perspectiva, obligando al lector a volver sobre el mismo texto para descodificarlo de nuevo (González Millán 1991: 15). Las versiones castellanas llevadas a cabo por el propio Cunqueiro no hacen sino intensificar estas estrategias narrativas ampliando, precisamente, el espacio del peritexto. Los microtextos añadidos en la "auto-traduction (re)créatrice" manifestarían, pues, un doble modo de actuación: el de la trangresión y el de la sobreestructuración, dando como resultado un proceso de organización textual extraordinariamente complejo. 
Pensemos, por ejemplo en el apéndice "Noticias varias de la vida de don Merlín, mago de Bretaña", un peritexto que, pese a estar situado en los márgenes, deviene esencial en la configuración general de la obra.

En "Noticias varias..." se narran, de acuerdo a uno de los tipos de extensiones temáticas más comunes aquella que consiste en la continuación analéptica del relato, las peripecias relativas al nacimiento y a las andanzas del joven Merlín.

En primer lugar, llama la atención el cambio de narrador que se produce en este nuevo microtexto. Frente al narrador autodiegético que aparece en el resto de la obra (el criado Felipe de Amancia que recuerda sus tiempos mozos al lado del mago Merlín, retirado en tierras de Miranda), en "Noticias varias" nos encontramos con un narrador heterodiegético que se corresponde implícitamente con un "yo" autorial. Este narrador da paso a una nueva voz, la del cronista inglés Mister Craven quien "le anunció a Felipe que venía a Miranda desde Rennes de Bretaña por establecer si don Merlín, en sus vacaciones gallegas había tenido descendencia" (Merlín-b: 167). Esta escena final supone una importantísima reorganización de la estructura narrativa de la novela. Así, frente al original gallego, presentado como el relato de las memorias de Felipe de Amancia dirigidas a un lector implícito, la versión castellana aparece como un intercambio de noticias entre Felipe y Craven.

Dentro del proceso de desintegración del estatuto de la voz narrativa y de la desestabilización diegética que se produce con la incorporación de este microtexto debe entenderse también la tensión que la voz de Craven genera al moverse entre la autoridad de la crónica histórica y el talante abiertamente paródico.

Así, Merlín, que en el discurso de Felipe de Amancia se ensalza como una figura perteneciente al ámbito de lo mítico y de lo prodigioso, aparece frecuentemente bajo el prisma de lo vulgar y cotidiano en el relato de Craven. Este procedimiento de desvaloración o trivialización de las motivaciones del héroe novelesco se observa, por ejemplo, en el siguiente pasaje en el que Mister Craven niega la castidad del mago:

(27) Y eso de la continencia por filósofo sería ahora de viejo, que de mozo y en las cortes, tu amo desenvainaba fácil

(Merlín-b: 169)

La tendencia a la desestabilización semántica que exhibe todo el microtexto analizado llega a uno de sus momentos límite cuando se nos explican los motivos del retiro de Merlín y doña Ginebra a Miranda:

(28) Y porque iba el que pasó a ser amo tuyo algo fatigado del mundanal ruido, y porque con la Revolución de Francia se quedara doña Ginebra sin las rentas [...] acordaron ambos retirarse a esperar mejores tiempos a Miranda

(Merlín-b: 191)

Esta información contrasta con la que nos había ofrecido Felipe de Amancia en el texto central:

(29) Decían que era [Dona Ginebra] viuda dun grande rei que morréu na guerra, e que tivo noticia por un corvo cando estaba en Miranda probando un peite de ouro

(Merlín: 19) 
Además, el apéndice "Noticias varias" funciona como intensificador de la dinámica intertextual característica del autor.

La imitación temática y estilística del subgénero de la novela itinerante, que floreció en las letras españolas durante los siglos XVI y XVII, es fácilmente identificable:

(30) Determinó el joven Merlín pasar de Madrid a Toledo, e iba muy seguro yendo a ciudad tan atareada de demonios, judíos, brujería y ciencias ocultas, porque en una posada, en Medina del Campo, había comprado a Isaac Zifar el nombre secreto de Toledo [...] en Madrid tomara trato Merlín con un caballero napolitano, [...] que venía a España a intrigar contra el señor virrey de Nápoles cerca del valido del Rey Católico, que lo era a la sazón el señor duque de Lerma [...] Llegó a Toledo Merlín, y asegurado por el duque de Lerma se vistió de gala y fue a llevarle al valido las cartas reservadas que traía, y preguntándole el duque por el viaje, no dejó don Merlín de contarle lo sucedido en Illescas. Dijo el duque de Lerma que sería burla de vagabundos y pícaros, y se rió, y le dijo que a la tarde siguiente podía venir a refrescarse a un cigarral, en el que un sobrino hacía fiesta

(Merlín-b: 185)

La recreación de subgéneros pertenecientes a los Siglos de Oro es frecuente en las obras castellanas de Cunqueiro. La preferencia por estos modelos no es, a nuestro entender, ajena a la influencia que sobre el autor ejerció la figura de Cervantes. En este sentido, la elección del subgénero sobre el que se realiza la imitación en el apéndice ejemplifica con especial nitidez el distanciamiento que se produce entre el original gallego y su versión castellana: el peritexto añadido tiene lugar desde la asunción de la tradición literaria de la lengua de llegada.

El dualismo de actitudes y valores que comporta el bilingüismo en la creación literaria se hace pues patente en esta autotraducción-recreación que da como resultado un híbrido cultural: desde el sistema gallego, Cunqueiro galleguiza el mito de Merlín echando mano de recursos propios de la tradición oral; desde el sistema castellano, nuestro escritor castellanizará la figura del mago, recurriendo a modelos literarios hipercodificados del barroco. La propia localización, Madrid, Toledo, así como la alusión a hechos y personajes históricos como el Duque de Lerma contribuirán explicitamente a la castellanización del mito artúrico.

Como podemos comprobar, las modificaciones en la versión son de tal calado que permiten hablar de una doble transformación idiomática e hipertextual.

Enfocada de este modo, la relación entre el texto gallego y castellano se presenta ya no como la de la tradicional dependencia jerárquica entre original y traducción sino como la que se establece entre simples variantes que constituyen fragmentos de un único macrotexto. Esta visión se enfrenta de lleno con la común tendencia de la crítica, que acostumbra a considerar la versión castellana como una obra cualitativamente superior ${ }^{6}$.

En la valoración de la "auto-traduction (re)créatrice" como obra autónoma influye, sin duda, no solo la transformación textual sino también el contexto sociológico en el que se desarrollan los pares de lenguas implicados. En ese sentido y siguiendo la terminología empleada por Grutman (2011), la traducción del gallego al castellano debe verse como un intercambio asimétrico en el que el autor, no simplemente bilingüe, sino diglósico, efectúa una "autotraducción vertical" que va "río arriba", de la lengua baja a la lengua alta. 
La capacidad del sistema literario español para fagocitar las culturas periféricas estatales se hace evidente, independientemente del carácter opaco o transparente de $^{7}$ la autotraducción. De hecho, la recepción de la obra cunqueiriana presenta perfiles confusos. Así nos encontramos ante dos actitudes contrapuestas: la de aquellos que consideran a Cunqueiro como perteneciente al sistema castellano, obviando su obra gallega, y la de quienes, conscientes del bilingüismo de su obra, lo perciben como un representante de una literatura regional que el sistema fuerte acoge en su seno.

\section{Conclusión}

Como vemos, el estatuto del texto autotraducido es enormemente ambigüo, puesto que, en la práctica, puede suplantar al original erigiéndose como un producto jerárquicamente superior debido a la labor de ampliación y perfeccionamiento de la simetría estructural del relato. Sin embargo, no debemos olvidar aquí que la idea de "segundo original" defendida, entre otros, por Oustinoff (2003) minimiza las marcas de la operación traductora, las estrategias propias de ese "enunciador segundo", de esa voz "distinta" que, como indicábamos al comienzo de nuestro trabajo, evidencia la existencia de un modelo de enunciación característico de la traducción.

Como hemos podido comprobar, en las versiones cunqueirianas se produce una operación de sustitución lingüística y cultural, de "traducción subinterpretada" (Fitch 1985: 111-125) que manifiesta tanto una fuerte tendencia a la disimilación, a la preservación "literal y literaria" del heterolingüismo; como a la asimilación, de acuerdo a una estrategia en la que se prima la "aceptabilidad" en la cultura meta. Las dos fuerzas opuestas, llevadas al extremo por un autor-traductor liberado del fantasma de la invisibilidad, acaban por ofrecer un texto excesivamente heterogéneo, marcado por "ethos" de signo muy diverso.

Además de la operación de transcripción, orientada hacia la microestructura, en las versiones analizadas se observa también la modalidad de "traducción suprainterpretada" (Fitch 1985: 111-125), aquella que manifiesta un proceso de sustitución de la macroestructura del texto transformando literariamente el original. Sin embargo, no nos encontramos tampoco aquí ante un ejercicio global, es decir ante "la repetición de un proceso", tal como apunta Fitch para diferenciar la autotraducción de la traducción clásica. De hecho, los desajustes diegéticos y enunciativos que produce la inclusión de peritextos en la versión castellana acaban por poner en cuestión la coherencia interna de la obra. A esto debe añadirse la apertura hacia los moldes genéricos de la tradición literaria española, lo que evidencia un proceso de aclimatación endógena.

Esta suma de factores nos lleva a cuestionar la concepción de autotraducciones cunqueirianas bien como texto jerárquicamente superior, bien como "segundo original", puesto que una concepción macrotextual, de acuerdo a la cual el texto de partida y de llegada se concebirían como variantes no intercambiables de un único texto hipotético, obviaría las características estrictamente ligadas tanto a esa "enunciación segunda" propia de la tradución como al ejercicio de deriva hipertextual que supone la recreación del original entendido como hipotexto. Parece, pues, pertinente establecer entre las versiones una escala que, sin olvidar la necesidad de contemplar el conjunto de las variantes, distinga la coherencia interna del primer original. 
Probablemente esta característica se manifieste no solo en las autotraducciones de Cunqueiro sino, en general, en las de la mayoría de escritores peninsulares en situación de bilingüismo endógeno; ahora bien, resulta innegable que en el caso de Cunqueiro se da así mismo una clara voluntad de proyección como escritor "biliterario", es decir aquel que se define, tal y como apunta Durišin (1993), como escritor que crea para dos literaturas o que crea para una literatura y traduce para la otra ${ }^{8}$.

A diferencia de los fenómenos de purgación léxica y semántica de Beckett o Nabokov, escritores que parecen buscar en la autotraducción un lenguaje vacacional que los aleje de lo materno, Cunqueiro ejerce la práctica bilingüe escribiendo sin renunciar a su origen. En este sentido, las autotraducciones sirven también para dar cuenta con especial nitidez de las normas y valores que caracterizan a las novelas escritas originariamente en castellano. Podemos comprobar así cómo el estilo en esta lengua es debido en gran parte a una labor consciente de metatraducción que busca la minorización de la lengua mayor por medio de la influencia del vernáculo. Así mismo, en el proceso de autotradución se puede observar la reflexión sobre las diferentes identidades con las que convive el escritor.

La asunción de la bicefalia, sitúa pues a obras como las de Cunqueiro -realizadas, no lo olvidemos, "río arriba", desde una clara situación de diglosia que evidencia una "subordinación colonial" (Casanova 2002)- en un "sistema intermediario" (Lambert 1987) que, al potenciar la interferencia de elementos entre dos literaturas, dificulta extraordinariamente la determinación misma del estatuto de ambos textos y, por lo tanto, su recepción. Las autotraduciones cunqueirianas vendrían, pues, a reclamar una concepción del espacio literario peninsular, marcado tradicionalmente por una concepción en exceso rígida de las fronteras lingüísticas y políticas, como un ámbito más amplio y complejo.

\section{NOTAS}

1. Como ha indicado Dasilva (2012: 96), buena parte de los poemas incluidos en Elegías y canciones (1940) fueron difundidos en gallego antes de su publicación en castellano.

2. A pesar de que la edición española presenta a Cunqueiro como único traductor, debemos indicar el carácter de "pseudotradución" o "tradución alógrafa", tal y como es denominada por Dasilva (2011: 46) que presenta la versión española de As crónicas do Sochantre. Así, la correspondencia entre el propio Cunqueiro y Francisco Fernández del Riego, amigo e impulsor de la editorial Galaxia fundamental en el proceso de recuperación de la cultura gallega en el franquismo, indica que es este último el responsable del trasvase lingüístico. Con todo, consideramos que la edición española es también relevante para el análisis que nos ocupa puesto que el autor participa en el proceso, supervisando la versión y proponiendo el añadido del peritexto "Epílogo para Bretones" y ciertas modificaciones en el texto de los Dramatis personae.

3. Las referencias a las obras gallegas Merlín e familia, As crónicas do Sochantre y Si o vello Sinbad volvese ás illas remiten a la edición siguiente: Álvaro Cunqueiro. Obra en galego completa. Narrativa. Vol. II, Vigo, Galaxia, 1982. Las referencias a Xente de aqui e acolá corresponden a la $7^{\mathrm{a}}$ edición publicada en Galaxia (1990). En relación con las versiones castellanas, las citas de Merlín y familia son de la $3^{a}$ edición de Destino (1991), las de Las crónicas del Sochantre pertenecen a la edición de la editorial AHR (1959) y las de La otra gente a la 2a edición de Destino (1991). Las abreviaturas con las que se designan las obras son las siguientes: Merlín e Familia e outras historias: Merlín; Merlín y familia: Merlín-b; Si o vello Sinbad volvese ás illas: Sinbad; Cuando el viejo Sinbad vuelva a las islas: Sinbad-b; Xente de aquí e acolá: Xente; La Otra gente: Gente; As crónicas do Sochantre: Sochantre; Las crónicas del Sochantre: Sochantre-b.

4. Así, por ejemplo, Guillén (1985: 343) cita como ejemplos de esta tendencia al tecnicismo o al refinamiento los juegos francófilos de Swinburne y Oscar Wilde o las páginas más empalagosas del último Nabokov. 
5. El concepto de metatraducción es empleado por George Steiner (1995: 15-51) para designar la influencia que ejerce una de las lenguas del escritor multilingüe sobre el resto. La creación en otros idiomas sería pues una suerte de traducción desde la lengua propia.

6. A este respecto resulta significativa la apreciación de la estudiosa Pérez-Bustamante (1991: 14):

"Cuando Cunqueiro traduce la versión gallega original, la castellana es más larga y manifiesta siempre, en las adiciones y redistribuciones del texto primitivo, una decidida voluntad de perfeccionamiento de la simetría estructural del relato, lo cual se revela sumamente significativo. Las mayores diferencias se dan entre los dos Merlines, y así comenta con toda razón Carballo Calero (1975: 280-281) que el segundo Merlín es una novela mucho más acabada, plena y "suficiente" que el primero, como, por lo demás salta a la vista. En menor medida, lo cual es a la vez síntoma de que el autor le tomó mejor el pulso a su arte, esto es aplicable a las otras novelas, de manera que, en realidad, el corpus elegido, en castellano, es siempre original, bien en la primera instancia, bien por la variaciones introducidas sobre la versión gallega".

7. Dasilva (2011) propone distinguir la autotraducción transparente, entendida como aquella que explicita su condición mediante informaciones paratextuales y la "autraducción opaca", aquella que no ofrece ningún dato acerca de su carácter de obra traducida.

8. Disiento en este aspecto del enfoque defendido por Dasilva, quien indica "a resolta vontade de Cunqueiro de formar parte, esencialmente, da literatura galega” (Dasilva 2012: 94). A este respecto, puede consultarse mi trabajo (Rodríguez Vega: 2001) en el que analizo las declaraciones de Cunqueiro en relación con su adscripción lingüística y literaria o el de Forcadela (2009: 150) en el que se observa el abandono final de la práctica de la novela en lengua gallega como señal de la ambigua posición de Cunqueiro en relación con el identitarismo nacional.

\section{REFERENCIAS}

Berman, Antoine (1985): La Traduction et la lettre ou l'auberge du lointain. Mauzevin: Trans Europ-Repress.

CARballo, Ricardo (1975): Historia da literatura galega contemporánea. Vigo: Galaxia.

Casanova, Pascale (2002): Consécration et accumulation de capital littéraire. La traduction comme échange inégal. Actes de la recherche en sciences sociales. 144(3):7-20.

Dasilva, Xosé Manuel (2009): Autotraducirse en Galicia: ¿bilingüismo o diglosia? Quaderns. 16:143-156.

Dasilva, Xosé Manuel (2010): La autotraducción vista por los escritores gallegos. In: Enric Gallén, Francisco Lafarga y Luis Pegenaute, eds. Traducción y autotraducción en las literaturas ibéricas. Bern: Peter Lang, 265-279.

Dasilva, Xosé Manuel (2011): La autotraducción transparente y la autotraducción opaca. In: Xosé Manuel Dasilva y Helena TANQueiro, eds. Aproximaciones a la autotraducción. Vigo: Editorial Academia del Hispanismo, 45-67.

Dasilva, Xosé Manuel (2012): Compromiso lingüístico e autotradución en Álvaro Cunqueiro. Grial. 194:94-103.

Ďurišin, DionÝz (1993): Notions et principes. Trad. Alena Anettova. Communautés interlittéraires spécifiques. Bratislava: Académie Slovaque des Sciences.

Fitch, Brian T. (1983): L'intertextualité interlinguistique de Beckett: la problématique de la traduction de soi. Texte. 2:85-100.

Fitch, Brian T. (1985): The status of Self-Translation. Revue de critique et de théorie littéraire. 4:111-125.

Forcadela, Manuel (2009): A mecánica da maxia. Ficcion e ideoloxía en Álvario Cunqueiro. Vigo: Galaxia.

GonzÁlez Millán, Xoán (1991): Álvaro Cunqueiro: os artificios da fabulación. Vigo: Galaxia. Grutman, Rainier (1997): Des langues qui résonnent. L’hétérolinguisme au XIXe siècle québécois. Québec: Fides.

GRUTMAN, Rainier (2007): L'écrivain bilingue et ses publics: une perspective comparatiste. In: Axel Gasquet y Modesta Suárez, eds. Écrivains multilingues et écritures métisses. L'hospitalité des langues. Clermont-Ferrand: Presses Universitaires Blaise Pascal, 31-50. 
GrUtman, Rainier (2009): La autotraducción en la galaxia de las lenguas. Quaderns. 16:123-134. Grutman, Rainier (2011): Diglosia y autotraducción vertical. In: Xosé Manuel Dasilva y Helena TANQUeiro, eds. Aproximaciones a la traducción. Vigo: Editorial Academia del Hispanismo, 69-92.

Guillén, Claudio (1985): Entre lo uno y lo diverso. Barcelona: Crítica.

LAMBERT, José (1987): Un modèle descriptif pour l'étude de la littérature. La littérature comme système complexe. Contextos. 5(9):47-67.

López Gay, Patricia (2008): La autotraducción literaria: traducibilidad, fidelidad, visibilidad. Análisis de las traducciones de Jorge Semprún y Agustín Gómez Arcos. Tesis de doctorado no publicada. Universitat Autònoma de Barcelona-Université Paris 7.

Meschonnic, Henri (1973): Pour la Poétique II. Epistémologie de l'écriture, poétique de la traduction. París: Gallimard.

Meschonnic, Henri (1989): La rime et la vie. París: Editions Verdier.

Oustinoff, Michaël (2001): Bilinguisme d'écriture et auto-traduction (Julien Green, Samuel Beckett, Vladimir Nabokov). París: L'Harmattan.

Oustinoff, Michaël (2003): La traduction. París: Presses Universitaires de France.

PARCERISAs, Francesc (2007): Idéologie et autotraduction entre cultures asymétriques. Atelier de Traduction. 7: 99-105.

Parcerisas, Francesc (2009): De l'asymétrie au degré zéro de l'autotraduction. Quaderns. 16:117-122.

Parcerisas, Francesc (2011): Autotraducción y lógica moral. In: Xosé Manuel Dasilva y Helena TANQueiro, eds. Aproximaciones a la autotraducción. Vigo: Editorial Academia del Hispanismo, 165-173.

Pérez-Bustamante, Ana Sofía (1991): Las siete vidas de Álvaro Cunqueiro. Cádiz: Servicio de Publicaciones de la Universidad de Cádiz.

SAntoyo, Julio César (2010): Autotraducciones intrapeninsulares: motivos históricos, razones actuales. In: Enric Gallén, Francisco Lafarga y Luis Pegenaute, eds. Traducción y autotraducción en las literaturas ibéricas. Bern: Peter Lang, 365-380.

TANQUeIro, Helena (1999): Un traductor privilegiado: el autotraductor. Quaderns. 3:19-27.

TANQueiro, Helena (2002): Autotraduçao: autoridade, privilégio e modelo. Tesis de doctorado no publicada. Barcelona: Universitat Autònoma de Barcelona.

R. Vega, Rexina (2001): Un escritor en contexto diglósico: a práctica bilingüe de Álvaro Cunqueiro. In: Dieter Kremer. Actas del $6{ }^{\circ}$ Congreso Internacional de Estudios Galegos. (Un século de estudios galegos: Galicia fóra de Galicia, La Habana, 17-21 de abril de 2000). Sada: Galizien-Zentrum der Universität Trier / Ediciós do Castro, 367-376.

Steiner, George (1995): Después de Babel. Aspectos del lenguaje y de la traducción. (Traducido por Adolfo Castañón) México: Fondo de Cultura Económica.

Suchet, Myriam (2009): Traduire Juan sin Tierra: une histoire d'ethos. In: Colloque Odyssée de la traductologie: Histoire de la traduction. Montréal, 27 de marzo de 2009. Consultado el 16 de septiembre de 2012, <http://www.uottawa.ca/associations/act.../Suchet_Traduire_ Juan_sin_Tierra.pdf>.

Toury, Gideon (1980): In Search of a Theory of Translation. Tel Aviv: Porter Institute for Poetics and Semiotics. 